\title{
The Diagnostic Utility of VibraTip for Distal Symmetrical Polyneuropathy in Type 2 Diabetes Mellitus
}

\author{
Nikolaos Papanas (D) - Kalliopi Pafili · Maria Demetriou • \\ Stella Papachristou - Soultana Kyroglou • Dimitrios Papazoglou • \\ Efstratios Maltezos
}

Received: August 6, 2019 / Published online: November 28, 2019

(C) The Author(s) 2019

\section{ABSTRACT}

Introduction: The aim of this study was to assess the performance of VibraTip, a device used to test a person's vibration perception during routine checks for peripheral neuropathy, against two thresholds of the Neuropathy Disability Score (NDS) for diagnosing distal symmetrical polyneuropathy (DSPN) in patients with type 2 diabetes mellitus (T2DM).

Methods: One hundred consecutive subjects with T2DM were enrolled in the study, of whom 54 were men. The mean age was 62.3 years, and the mean T2DM duration was 12.6 years. VibraTip was used at one foot site (on the pulp

Enhanced Digital Features To view enhanced digital features for this article go to: https://doi.org/10.6084/ m9.figshare.10564058.

N. Papanas $(\varangle) \cdot$ K. Pafili - M. Demetriou . S. Papachristou - S. Kyroglou - D. Papazoglou . E. Maltezos

Diabetes Centre-Diabetic Foot Clinic, Second Department of Internal Medicine, Democritus University of Thrace, Alexandroupolis, Greece e-mail: papanasnikos@yahoo.gr of the hallux; protocol A) and at three foot sites (pulp of the hallux and first and third metatarsal head; protocol B). NDS thresholds of $\geq 3$ and $\geq 6$ were used to establish the diagnosis of DSPN.

Results: Against the NDS $\geq 3$ threshold, VibraTip showed a very high sensitivity (91.3\%) and negative predictive value (NPV) (92\%) and a high specificity (85.2\%) with protocol $\mathrm{A}$, and a very high sensitivity (95.6\%) and NPV (96.1\%) and a very high specificity $(90.7 \%)$ with protocol B. Against the NDS $\geq 6$ threshold, VibraTip showed a very high sensitivity (100\%) and NPV $(100 \%)$ and a very high specificity $(95.2 \%)$ with protocol A, and very high sensitivity (100\%) and NPV (100\%) and very high specificity (96.8\%) with protocol B.

Conclusions: The diagnostic performance of VibraTip is very high in patients with T2DM, rendering it a very useful device as a screening tool, particularly for the exclusion of DSPN. VibraTip performs very well at both NDS thresholds, but particularly well at the NDS $\geq 6$ threshold. There appears to be no need to examine sites other than the hallux site with Vibratip.

Keywords: Clinical examination; Complications; Diabetic neuropathy; Diagnosis; Type 2 diabetes mellitus; Vibratip 


\section{Key Summary Points}

Distal symmetrical polyneuropathy (DSPN) needs to be diagnosed early in patients with type 2 diabetes mellitus, which requires larger-scale screening of patients and the development of new diagnostic tools.

We have examined the diagnostic performance of the pocket-sized portable device VibraTip for testing a person's vibration perception versus standardised clinical examination using the neuropathy disability score (NDS) at two thresholds: NDS $\geq 3$ (mild DSPN) and NDS $\geq 6$ (moderate DSPN), with the higher NDS threshold showing an increased risk for foot ulceration.

We also examined whether VibraTip used solely at the hallux is sufficient for diagnostic efficacy or whether the diagnostic yield can be improved by adding two more examination sites on the foot.

At both NDS thresholds, VibraTip yielded very high sensitivity and negative predictive values, high/very high specificity values and very low negative likelihood ratios. Overall, its diagnostic performance was very good.

The main utility of VibraTip appears to lie in the exclusion of DSPN.

The application of Vibratip to only the hallux is sufficient to ensure good diagnostic performance.

\section{INTRODUCTION}

Neuropathy remains one of the most common chronic complications of diabetes mellitus [1]. In practice, it usually manifests as distal symmetrical polyneuropathy (DSPN), a chronic, insidious, duration-dependent sensory or sensorimotor neuropathy $[1,2]$. DSPN is a cardinal aetiological factor for diabetic foot lesions, and it may also lead to chronic pain and other further complications $[1,3]$. To avoid these complications, timely diagnosis of DSPN is an absolute priority $[1,3]$. Clinical examination encompassing sensory and motor modalities remains the cornerstone of diagnosis in realworld settings [1, 3]. Efforts to improve the diagnosis of DSPN and facilitate larger-scale patient screening have resulted in the development of new tests $[3,4]$, such as the indicator test for sudomotor function (Neuropad [5]), the steel ball-bearing test [6], the automated nerve conduction study of the sural nerve $[7,8]$, VibraTip $[9,10]$, among others.

VibraTip (McCallan Medical Ltd., Nottinghamshire, UK) is a pocket-sized portable device for the diagnosis of DSPN through evaluation of vibration perception in the foot (primarily at the hallux) $[9,10]$. It has good reproducibility and is noted for its reliable diagnosis of DSPN, while it also shows agreement with the vibration perception threshold, as measured with a neurothesiometer and the $128 \mathrm{~Hz}$ tuning fork $[9,10]$. VibraTip has already been compared with the validated and standardised Neuropathy Disability Score (NDS) [11] in the clinical setting. However, in that study VibraTip was compared with the NDS threshold of $\geq 6$ [9], which is an excellent threshold for identifying feet at risk of ulceration [12], but less so for identifying persons with mild incipient DSPN, for which a threshold of $\geq 3$ would be useful [11]. Thus, the aim of this study was to examine: (1) the diagnostic performance of VibraTip against both of these NDS thresholds ( $\geq 3$ and $\geq$ 6 ) for the diagnosis of DSPN in persons with type 2 diabetes mellitus (T2DM); (2) whether the addition of two examination areas other than the hallux in the foot increases the diagnostic performance.

\section{METHODS}

One hundred prospective T2DM subjects (54 men) with a mean age of 62.3 years, mean T2DM duration of 12.6 years and mean glycated 
haemoglobin of $7.7 \%(61 \mathrm{mmol} / \mathrm{mol})$ who regularly attended our Diabetes Centre were included in the study. Exclusion criteria were pregnancy, lactation, B12 depletion, alcohol abuse and other causes of peripheral neuropathy.

The subjects were examined with VibraTip $[9,10]$ using two examination protocols: (1) at one foot site (on the pulp of the hallux; protocol A) and (2) at three foot sites (pulp of the hallux and first and third metatarsal head; protocol B). The device was placed in random order of activation/non-activation on the sites of examination [9]. With both protocols, the test result was considered to be abnormal if vibration was not felt in one or more sites.

The reference method for the diagnosis of DSPN was the NDS [11], a clinical score that has been developed to assess diabetic neuropathy The NDS is an established standardised and very widely used clinical examination score that assesses both sensory and motor modalities, both small and large nerve fibre function [11]. Its score (0-10) is very useful for the diagnosis and staging of DSPN. In our evaluation, we used two NDS thresholds: NDS $\geq 3$ (mild neuropathy) and NDS $\geq 6$ (moderate neuropathy) [11]. Examination with NDS was carried out by a physician blinded to the results of the VibraTip examination.

Sensitivity, specificity, positive predictive value (PPV), negative predictive value (NPV), positive likelihood ratio $(\mathrm{LR}+)$, negative likelihood ratio (LR-) and Youden's J were calculated using standard formulae.

The study was approved by the ethics committee of Democritus University of Thrace. It was carried out in accordance with the Helsinki Declaration of Human Rights of 1964, as revised in 2013 , and patients provided their informed consent.

\section{RESULTS}

Overall frequency of DSPN as assessed using the NDS threshold of $\geq 3$ (mild neuropathy) was $46 \%(n=46)$. Moderate DSPN, based on the NDS threshold of $\geq 6$ was diagnosed in 38 subjects (38\%).

Against the NDS threshold of $\geq 3$, VibraTip yielded $91.3 \%$ sensitivity and $85.2 \%$ specificity with protocol A and $95.6 \%$ sensitivity and 90.7\% specificity with protocol B (Table 1 ).

Against the NDS threshold of $\geq 6$, VibraTip yielded $100 \%$ sensitivity and $95.2 \%$ specificity with protocol A and 100\% sensitivity and 96.8\% specificity with protocol B (Table 2).

\section{DISCUSSION}

The results of this study show that the diagnostic performance of VibraTip for DSPN is very good compared to clinical examination based on the NDS. In particular, VibraTip exhibited a very high sensitivity, high/very high specificity, high PPV and very high NPV. These results are in agreement with those reported by Bowling et al. [9], but the latter only used the NDS $\geq 6$ threshold. By contrast, we used both the ND $\geq 3$ and ND $\geq 6$ thresholds, and the results confirm the diagnostic utility of VibraTip against both thresholds. This is an important result because the NDS $\geq 3$ threshold is the more valuable threshold for early detection of mild DSPN, while the NDS $\geq 6$ threshold has been proposed as being the more useful threshold for

Table 1 Diagnostic performance of VibraTip against the Neuropathy Disability Score threshold of $\geq 3$

\begin{tabular}{llllllll}
\hline VibraTip protocol $^{\mathbf{a}}$ & Sensitivity (\%) & Specificity (\%) & PPV (\%) & NPV (\%) & LR+ & LR- & Youden's $\boldsymbol{J}$ \\
\hline Protocol A & 91.3 & 85.2 & 84 & 92 & 6.2 & 0.1 & 0.765 \\
Protocol B & 95.6 & 90.7 & 89.8 & 96.1 & 10.3 & 0.05 & 0.863 \\
\hline
\end{tabular}

$L R+$ Positive likelihood ratio, $L R-$ negative likelihood ratio, $N C S$ nerve conduction study, $N P V$ negative predictive value, $P P V$ positive predictive value

${ }^{a}$ VibraTip was used at one foot site (on the pulp of the hallux) in protocol A and at three foot sites (pulp of the hallux and first and third metatarsal head) in protocol B 
identifying more severe DSPN with the risk of future diabetic foot ulceration [12]. VibraTip performed very well against both NDS thresholds, but particularly against the NDS $\geq 6$ threshold. Additionally, we report a satisfactorily high LR+ and, most importantly, an extremely low LR- (around 0), which suggests for the first time that the primary importance of VibraTip is to exclude DSPN. The very good diagnostic performance of the device is also reflected in the high Youden's $J$ values. Other researchers have demonstrated the good diagnostic yield of VibraTip in comparison with different modalities (neurothesiometer, $128 \mathrm{~Hz}$ tuning fork, Semmes-Weinstein monofilament, NeuroTip) [10, 13].

In a further step, we examined whether the use of additional examination sites on the foot (in addition to the pulp of the hallux) would improve the diagnostic performance of VibraTip. Our three-site examination protocol (pulp of the hallux, first and third metatarsal head) is similar to the five-site examination protocol (first, third and fifth metatarsal heads, hallux pulp, and dorsal surface of the hallux proximal to the nail fold) reported by Bracewell et al. [10]. Nevertheless, the latter authors did not compare the results of their five-site protocol with the hallux-only examination and they did not use the NDS for comparison [10]. In our study, the three-site examination protocol improved the $\mathrm{LR}+$ but conferred only very minute increases in the other diagnostic parameters. Hence, it would appear that it is generally not necessary to add two extra examination sites when using VibraTip and that examination of the hallux is sufficient for reliable results. Clearly, the simplicity and rapidity of the procedure increases when only the the hallux needs to be examined, adding to the value of Vibratip as a simple screening test.

A major strength of this study includes the utilisation of the validated NDS for reliable clinical examination and comparison. A further strength is the use of two NDS thresholds, one to detect mild DSPN and one to detect moderate DSPN [11]. We also specifically addressed the question of whether the addition of two examination sites in the foot would improve the diagnostic performance of VibraTip. An important limitation is the tertiary care setting, which explains the rather high frequency of DSPN in our series. Thus, caution is needed before generalising these results to the primary care setting. A further limitation is the small study population.

The clinical implications of these findings can be outlined as follows. VibraTip may be used to screen subjects with T2DM for DSPN. The triad of very high sensitivity and NPV together with very low LR - render it especially useful as a diagnostic tool to exclude DSPN; this holds true both for mild DSPN (NDS $\geq 3$ ) and moderate DSPN (NDS $\geq 6$ ) [11]. For this purpose, the advantages of the device (small size, easy use, no need for expert training) $[9,10]$ are important. Of practical importance, examination of vibration perception on the hallux is sufficient, and there is virtually no need to examine other sites (such as the first and third metatarsal heads in the present study) with the Vibratip.

\section{CONCLUSIONS}

The diagnostic performance of VibraTip is very high in persons with T2DM, rendering it very useful as a screening tool, primarily for the exclusion of DSPN. VibraTip performs very well against both the NDS $\geq 3$ and NDS $\geq 6$ thresholds, but particularly against the NDS $\geq 6$ threshold. It is not necessary to examine sites other than the hallux with the Vibratip as the inclusion of addional sites in the examination provides only a very slight improvement in

Table 2 Diagnostic performance of VibraTip the Neuropathy Disability Score threshold of $\geq 6$

\begin{tabular}{llllllll}
\hline VibraTip protocol & Sensitivity (\%) & Specificity (\%) & PPV (\%) & NPV (\%) & LR+ & LR- & Youden's $J$ \\
\hline Protocol A & 100 & 95.2 & 92.7 & 100 & 20.8 & 0 & 0.952 \\
Protocol B & 100 & 96.8 & 95 & 100 & 31.25 & 0 & 0.968 \\
\hline
\end{tabular}


diagnostic performance. Overall, VibraTip appears to merit further use in clinical practice [14], which will promote larger-scale patient screening and improve early DSPN diagnosis [3, 15-17], in harmony with the need for a quick and reliable comprehensive foot examination [18].

\section{ACKNOWLEDGEMENTS}

The authors thank the participants of the study.

Funding. No funding or sponsorship was received for this study or publication of this article.

Authorship. All named authors meet the International Committee of Medical Journal Editors (ICMJE) criteria for authorship for this article, take responsibility for the integrity of the work as a whole, and have given their approval for this version to be published.

Disclosures. Nikolaos Papanas has been an advisory board member of TrigoCare International, Abbott, AstraZeneca, Elpen, MSD, Novartis, Novo Nordisk, Sanofi-Aventis and Takeda; has participated in sponsored studies by Eli Lilly, MSD, Novo Nordisk, Novartis and Sanofi-Aventis; received honoraria as a speaker for AstraZeneca, Boehringer Ingelheim, Eli Lilly, Elpen, Galenica, MSD, Mylan, Novartis, Novo Nordisk, Pfizer, Sanofi-Aventis, Takeda and Vianex; and attended conferences sponsored by TrigoCare International, AstraZeneca, Boehringer Ingelheim, Eli Lilly, Novartis, Novo Nordisk, Pfizer and Sanofi-Aventis. Kalliopi Pafili, Maria Demetrio, Stella Papachristou, Soultana Kyroglou, Dimitrios Papazoglou and Efstratios Maltezos have nothing to disclose.

Compliance with Ethics Guidelines. The study was approved by the ethics committee of Democritus University of Thrace. It was carried out in accordance with the Helsinki Declaration of Human Rights of 1964, as revised in 2013, and patients provided their informed consent.
Data Availability. The datasets during and/ or analyzed during the current study are available from the corresponding author on reasonable request.

Open Access. This article is distributed under the terms of the Creative Commons Attribution-NonCommercial 4.0 International License (http://creativecommons.org/licenses/ by-nc/4.0/), which permits any noncommercial use, distribution, and reproduction in any medium, provided you give appropriate credit to the original author(s) and the source, provide a link to the Creative Commons license, and indicate if changes were made.

\section{REFERENCES}

1. Pop-Busui R, Boulton AJ, Feldman EL, et al. Diabetic neuropathy: a position statement by the American Diabetes Association. Diabetes Care. 2017;40: 136-54.

2. Ziegler D, Papanas N, Vinik AI, Shaw JE. Epidemiology of polyneuropathy in diabetes and prediabetes. Handb Clin Neurol. 2014;126:3-22.

3. Bönhof GJ, Herder C, Strom A, Papanas N, Roden M, Ziegler D. Emerging biomarkers, tools, and treatments for diabetic polyneuropathy. Endocr Rev. 2019;40:153-92.

4. Papanas N, Ziegler D. New vistas in the diagnosis of diabetic polyneuropathy. Endocrine. 2014;47: 690-8.

5. Papanas N, Boulton AJ, Malik RA, et al. A simple new non-invasive sweat indicator test for the diagnosis of diabetic neuropathy. Diabet Med. 2013;30: $525-34$.

6. Papanas N, Gries A, Maltezos E, Zick R. The steel ball-bearing test: a new test for evaluating protective sensation in the diabetic foot. Diabetologia. 2006;49:739-43.

7. Pafili K, Maltezos E, Papanas N. NC-stat for the diagnosis of diabetic polyneuropathy. Expert Rev Med Dev. 2017;14:251-4.

8. Chatzikosma G, Pafili K, Demetriou M, Vadikolias K, Maltezos E, Papanas N. Evaluation of sural nerve automated nerve conduction study in the diagnosis of peripheral neuropathy in patients with type 2 diabetes mellitus. Arch Med Sci. 2016;12:390-3. 
9. Bowling FL, Abbott CA, Harris WE, Atanasov S, Malik RA, Boulton AJ. A pocket-sized disposable device for testing the integrity of sensation in the outpatient setting. Diabet Med. 2012;29:1550-2.

10. Bracewell N, Game F, Jeffcoate W, Scammell BE. Clinical evaluation of a new device in the assessment of peripheral sensory neuropathy in diabetes. Diabet Med. 2012;29:1553-5.

11. Young MJ, Boulton AJ, MacLeod AF, Williams DR, Sonksen PH. A multicentre study of the prevalence of diabetic peripheral neuropathy in the United Kingdom hospital clinic population. Diabetologia. 1993;36:150-4.

12. Abbott CA, Carrington AL, Ashe H, et al. The NorthWest Diabetes Foot Care Study: incidence of, and risk factors for, new diabetic foot ulceration in a community-based patient cohort. Diabet Med. 2002;19:377-84.

13. Nizar H, Munro N, Nightingale P, Feher MD. Diagnostic accuracy of the VibraTip in detection of diabetic peripheral neuropathy. Br J Diabetes Vasc Dis. $2014 ; 14: 26-9$.
14. Goddard K, Vas P, Purves A, et al. Comparing the diagnostic accuracy of simple tests to screen for diabetic peripheral neuropathy: protocol for a crosssectional study. JMIR Res Protoc. 2018;7:e72.

15. Pafili K, Papanas N, Ziegler D. Neuropathy in diabetes: "one cannot begin it too soon". Angiology. 2018;69:752-4.

16. Migdalis I, Czupryniak L, Lalic N, Leslie RD, Papanas N, Valensi P. Diabetic microvascular complications. Int. J Endocrinol. 2018;2018:5683287.

17. Körei AE, Istenes I, Papanas N, Kempler P. Smallfiber neuropathy: a diabetic microvascular complication of special clinical, diagnostic, and prognostic importance. Angiology. 2016;67:49-57.

18. Woodbury MG, Sibbald RG, Ostrow B, Persaud R, Lowe JM. Tool for rapid \& easy identification of high risk diabetic foot: validation \& clinical pilot of the simplified 60 second diabetic foot screening tool. PLoS One. 2015;10:e0125578. 